\title{
EFFICIENT EYES DETECTION USING FAST NORMALISED CROSS-CORRELATION
}

\author{
N K Bansode ${ }^{1}$ and Dr P K Sinha ${ }^{2}$ \\ ${ }^{1}$ Department of Computer Engineering, College of Engineering, Pune. \\ nk_bansodeerediffmail.com \\ Centre for Development of Advanced Computing, Pune. \\ psinha@cdac.in
}

\begin{abstract}
Eye detection is important for finding locations of the face landmarks such as nose, ear and mouth on the face. Template matching and normalized cross correlation techniques are used for eye detection. The template matching is better than appearance and feature based method for eye detection in terms of accuracy. The experiment is performed on chufs database of face image for detection of eyes. The experimental results demonstrate the effectiveness of the template matching methods for eyes detection.
\end{abstract}

\section{KEYWORDS}

Image Processing, Cross-correlation, Template matching.

\section{INTRODUCTION}

In Computer Vision domain, one of the main development areas is related to the faces, either in static images or video sequence of images. The face detection and recognition have become important in the field of Computer Vision and Pattern Classification [1]. The face recognition have been used in various applications such as security, surveillance systems, human computer interfacing, suspect investigation. The task of eye detection plays very important role in the face detection and recognition. The preprocessing stage of face detection, recognition includes localisation and features extraction of facial components like eyes, nose and mouth etc. The eyes can be considered salient and relatively stable feature in comparison with other facial features.

Once face is detected, it is necessary to align faces for recognition. The face alignment is usually performed by position of other face landmarks with reference to the eye locations [2]. The performance of face recognition rate degrades rapidly when the face components alignment is not proper. This indicates that eye detection is very important in the face recognition system. However, eye detection remains a very challenging task due to several variations. The shape and appearance of eyes vary with identity, viewing direction, illumination condition, eye motion, and occlusion due to eyeglasses [3]. The objective of this work is to present effective and fast eye detection using normalized cross correlation technique.

\section{LITERATURE SURVEY}

Eye detection plays very important role in the alignment of a face in the proper position for face detection and recognition. Considering the position of the eyes, the distances of the other landmark points are calculated [4]. The methods for eye detection is divided into two main categories : active and passive approach. The active approach consists of focusing on the infrared light beam into the eyes. The cornea region reflects the beam back. This phenomenon produces high bright pupils in gray scale images including eye positions [5].

DOI : $10.5121 /$ sipij.2012.3605 
Signal \& Image Processing : An International Journal (SIPIJ) Vol.3, No.6, December 2012

Cameron Whitelam et al [6] proposed the spectral properties of the pupil near infrared illumination. Eyes are explored in the context of the different bands such as visible, multi spectral and short waves. The eyes on the face images captured under different bands and detected. It is used for the infrared illuminated images only. The passive approach can be further classified into three categories: template matching, appearance and feature based methods.

\subsection{Template Based Method}

In the template based method, a template consisting one of the feature of a face like eye is prepared. The eye template matching is used to search the image for the eyes on pixel by pixel basis. The accuracy of the matching is better as compared to the appearance and feature based methods for feature detection. The template matching is time consuming due to large search space [7]. In order to improve the efficiency of this method, cross correlation technique has been proposed that reduces the time required for matching template with the region of interest in the face image.

\subsection{Feature Based Method}

In this method, image feature characteristics such as edge, intensity and colour are used to identify some distinctive features of the eyes. The accuracy of the feature based method is less due to large variations in the feature space. The feature vector is compared with the image vector to find the presence of the feature in the image. Qiong Wang et al [8] used structure of the eye region as important cue for detecting the eye pair. The eye pair detection algorithm works on the whole gray-level image and homographic filter is applied to enhance the contrast of the dark region. The clustering and threshold techniques are used for different gray levels feature separations. The template matching with support vector machine is used for extracting the eyes. Kun Peng et al [9] presented combined approach of feature based method for detecting eye region and template based method for locating center of the iris. The size of eye template is estimated from the width of a face. The horizontal and vertical projections are used for estimation of face width and height.

S.Asteriadis et al [10] presented method for eye localization based on the geometrical information. Initially face is detected and bounding box enclosed on the face. The edge map of face image in the bounding box is extracted. The canny edge detector is used to produce the vector length and edge map for an image. The principal component analysis is applied to find the eigenvectors of training images. The similarity of weights with eye model is used to declare an eye presence.

\subsection{Appearance Based Method}

In a appearance based detection, features such as color, texture and shape can be analyzed. Generally appearance based method requires large amount of training data for representing eyes of different subjects, orientation and illumination conditions. The classifiers such as neural network and support vector machine are used to detect the eyes in the face image.

Chughoon Kim et al [11] described composite vector with biased discrimination analysis for eye detection. A pattern is represented by the set of variables which are called as primitive variables. The composite vector composed of a number of primitive variables and hybrid cascade detector is constructed for eye detection using haar like features in the early stage and composite biased discrimination analysis(C-BDA) in the latter stage. The C-BDA is derived from covariance of the composite vectors.

Zhou and Geng [12] presented an idea of the integral projection, variance projection and the generalized projection function and implemented hybrid projection function which is better than the variance and integral projection function. It requires each eye should be in separate window. 
Signal \& Image Processing : An International Journal (SIPIJ) Vol.3, No.6, December 2012

M. Hassaballah and Shun Ido [13] explored the method based on the entropy of the eye region. The center of eye region is too dark compared to the neighbouring regions. A score based on the entropy of eye and the darkness of an iris is used to detect the eye centre coordinates. A probability distribution of gray values can be estimated by counting the number of times each gray value occurs in the image and dividing by the total number of occurrence. In the eye region, the intensity values are unpredictable and correspondence to the high entropy. An iris is circular in shape and dark in colour. The sum of intensity values in a circle of radius $r$ around the center of each window is calculated. The structure of the paper as follows. Section 3 describes the methodology used to detect the eyes. In section 4 , implementation results and discussion are presented. Section 5 presents concluding remarks on the eye detection.

\section{METHODOLOGY}

In this section we outline the technique to perform eye detection. The proposed method is presented in Fig. 1. In this method we assume that input image consists of single face image. When the face is presented to eye detection system, Face image extracted from the background and face size is calculated. The detailed description of eyes detection system is given in the following steps.

\subsection{Preprocessing}

The preprocessing is essential for enhancement of the image required for the further processing. The colour image is converted into grey level image. The face image is separated from the background. The face top, bottom as well as left, right boundaries are used to locate face image.

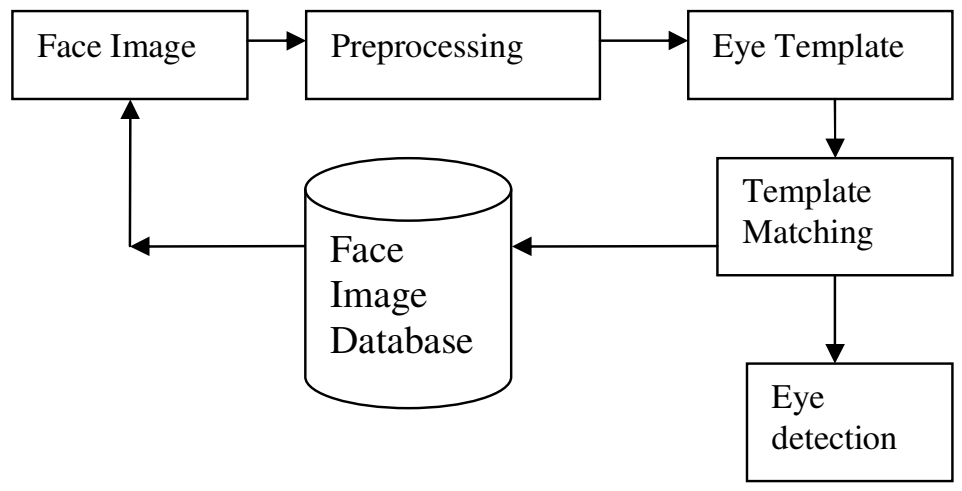

Fig. 1 Eyes Detection System

\subsection{Eye Detection}

Eye detection is performed by comparing the eye template with face image in order to detect the position of eye on the face. The normalized cross correlation is calculated between template and face image as given in equation (1). The normalized cross correlation gives maximum value of correlation coefficient when the input template matches exactly with the region on the face image. The correlation is statistical technique [14] that can show whether two or more variables are strongly related. The degree of relationship between the variables under consideration is measured through the correlation analysis [15]. The measure of correlation is called the coefficient of correlation or correlation index. The correlation analysis refers to the techniques used in measuring the closeness of the relationship between the variables [16]. The coefficient of correlation is one of the most widely used and observed statistical measure [17-18] 


\subsection{Template Matching}

In the eye template matching method, eye template is prepared for the eye detection on the face image. The templates used for eyes detection are shown in fig 2.

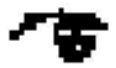

(a)

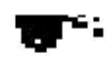

(b)

Fig. 2 Eye Template a) Left b) Right

$\gamma(u, v)=\frac{\sum_{x, y}\left[f(x, y)-f^{-} u, v\right]\left[t(x-u, y-v) t^{-}\right]}{\left\{\sum_{x, y}\left[f(x, y)-f^{-} u, v\right]^{2} \sum_{x, y}\left[t(x-u, y-v) t^{-}\right]^{2}\right\}^{0.5}}$

Where $f(x, y)$ is image of size $M \times N$

$t(u, v)$ is template $m \times n$

$f_{u, v}{ }^{-} \quad$ mean of $f(x, y)$ in the region under the template.

$t \quad$ mean of the template.

\section{RESUlt AND DisCUSSION}

In this section we represent the experimental results of eye template matching using cross correlation techniques. The proposed method has been tested by images from chufs face database [19]. The experimental results show that, the template matching using the normalized cross correlation method is accurate and fast. The graph in fig. 5 shows the time required to detect right eye is more compare to the left eye. We found that the average time required to detect left eye on the face image is 1.549 seconds and for right eye 1.950 seconds for 20 faces. The time for right eye detection is more due to the position of the eye on the face.

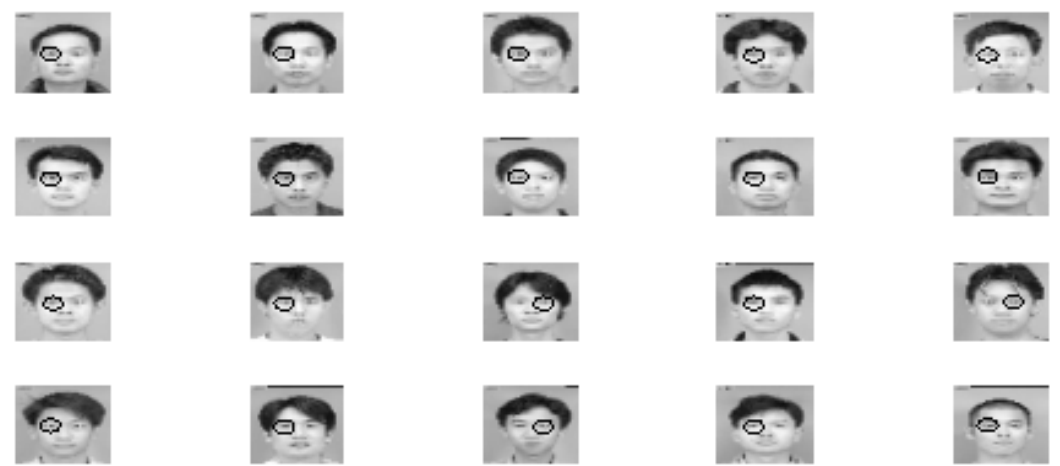

Fig. 3 Left Eye Detection 
Signal \& Image Processing : An International Journal (SIPIJ) Vol.3, No.6, December 2012

Table 1. Time for left eye detection

\begin{tabular}{|l|l|l|l|l|l|}
\hline $\begin{array}{l}\text { Sr. } \\
\text { No. }\end{array}$ & No of Faces & Time(sec) & $\begin{array}{l}\text { Sr. } \\
\text { No. }\end{array}$ & No of Faces & Time(sec) \\
\hline 1 & 1 & 0.35 & 11 & 11 & 1.53 \\
\hline 2 & 2 & 0.60 & 12 & 12 & 1.70 \\
\hline 3 & 3 & 0.74 & 13 & 13 & 1.79 \\
\hline 4 & 4 & 0.82 & 14 & 14 & 1.89 \\
\hline 5 & 5 & 1.09 & 15 & 15 & 2.03 \\
\hline 6 & 6 & 1.00 & 16 & 16 & 2.21 \\
\hline 7 & 7 & 1.15 & 17 & 17 & 2.32 \\
\hline 8 & 8 & 1.23 & 18 & 18 & 2.41 \\
\hline 9 & 9 & 1.37 & 19 & 19 & 2.58 \\
\hline 10 & 10 & 1.45 & 20 & 20 & 2.72 \\
\hline
\end{tabular}

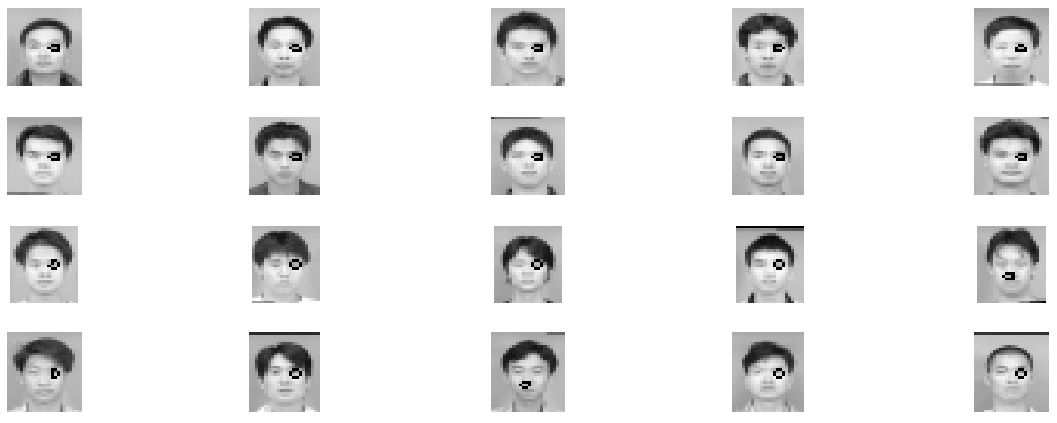

Fig. 4 Right Eye Detection

Table 2. Time for right eye detection

\begin{tabular}{|l|l|l|l|l|l|}
\hline $\begin{array}{l}\text { Sr. } \\
\text { No. }\end{array}$ & No of Faces & Time(sec) & $\begin{array}{l}\text { Sr. } \\
\text { No. }\end{array}$ & No of Faces & Time(sec) \\
\hline 1 & 1 & 0.37 & 11 & 11 & 2.01 \\
\hline 2 & 2 & 0.65 & 12 & 12 & 2.20 \\
\hline 3 & 3 & 0.79 & 13 & 13 & 2.38 \\
\hline 4 & 4 & 0.84 & 14 & 14 & 2.48 \\
\hline 5 & 5 & 1.12 & 15 & 15 & 2.67 \\
\hline 6 & 6 & 1.16 & 16 & 16 & 2.82 \\
\hline 7 & 7 & 1.31 & 17 & 17 & 2.99 \\
\hline 8 & 8 & 1.53 & 18 & 18 & 3.20 \\
\hline 9 & 9 & 1.66 & 19 & 19 & 3.37 \\
\hline 10 & 10 & 1.85 & 20 & 20 & 3.52 \\
\hline
\end{tabular}




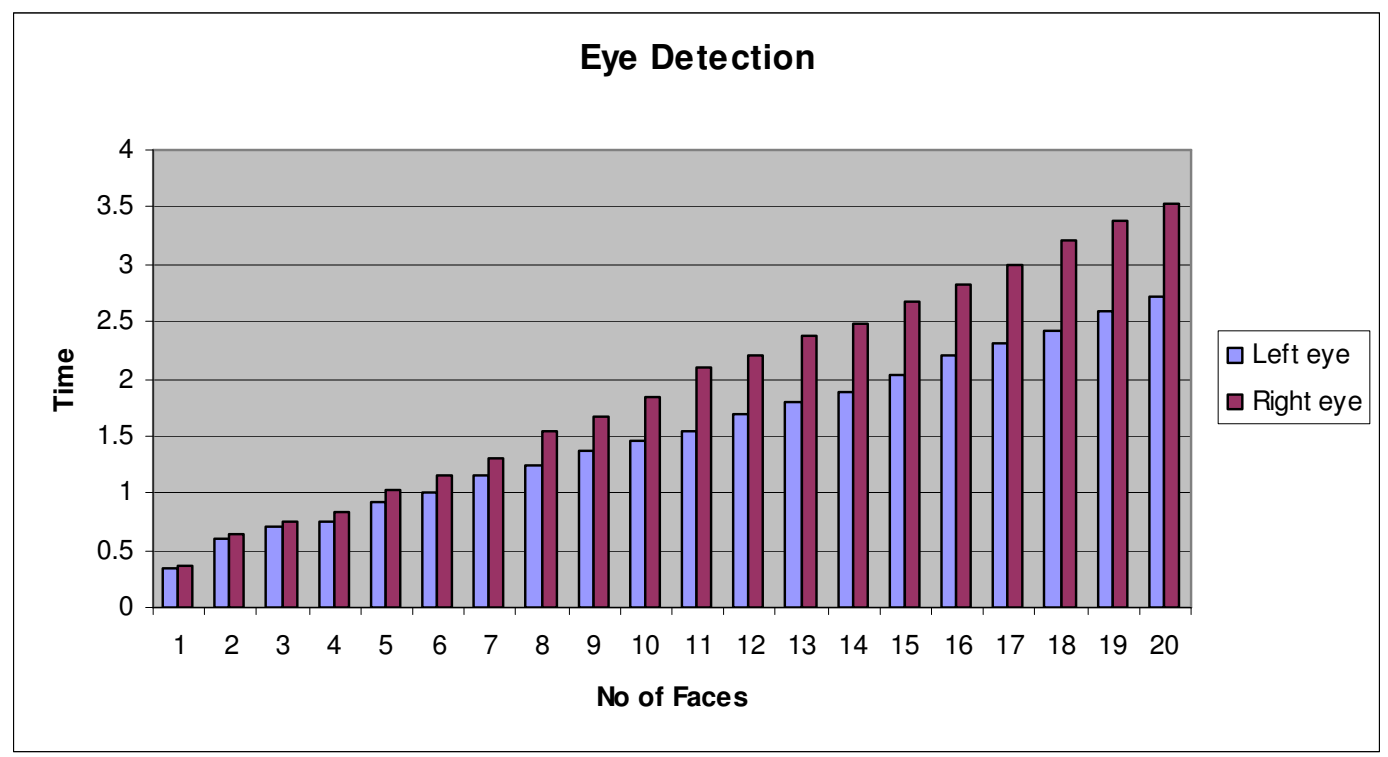

Fig. 5 Time required for Eyes Detection

\section{Conclusions}

We have presented a study on the problem of the eyes detection in a face image. The efficiency of template matching method is presented. The eye detection plays important role in the locations of the nose, ear and mouth. The proposed method has been tested by images from chufs face database. We found that the template matching is accurate method for eyes detection.

\section{REFERENCES}

[1] Manal Abdullah, Majda Wazzan, Shar Bo-saeed (2012) "Optimizing Face Recognition Using PCA" International Journal of Artificial Intelligence \& Applications (IJAIA) Vol. 3 No. 2 March 2012

[2] Hazem M. EI-Bakry (2008) "A New Implementation of PCA for fast Face Detection", World Academy of Sciences Engineering and Technology, 2008

[3] Chunghoon Kim, Matthew Truk 2012, "A New Biased Discriminant Analysis Using Composite Vectors for Eye Detection", IEEE Transaction on System Man and Machine

[4] Yi sun ,"Eye Pair Detection Using Geometric Surface Labeling and Posterior Probability Maximization", - Technical Report.

[5] Bruno de Brito Leite, Luciana Ribeiro Veloso, "A Learning-based Eye Detector- Coupled with Eye Candidate Filtering and PCA Features"

[6] Cameron Whitelam, Zain Jafari ,Thirimathces Bourtai (2010) "Multi Spectral Eye Detection: A Preliminary Study", International conference on Pattern Recognition 2010.

[7] Mihir Jain, Suman Mitra, Naresh "Eye Detection and Face Recognition Using Line Edge Map"

[8] Qiong Wang, Jingyu Yang (2006), "Eye Detection in facial Image with Unconstrained Background",Journal of Pattern Recognition Research 2006.

[9] Kun Peng, Liming Chen (2005) "A Robust Algorithm for Eye Detection on Gray Intensity Face without Spectacles", Journal of Computer Science and Technology vol. No. 5 No.3, October 2005.

[10] S. Asteriandis, N Nikoladis, A.Hajdu I. Pitas. "An Eye Detection Algorithm Using Pixel to Edge Infromation". 
Signal \& Image Processing : An International Journal (SIPIJ) Vol.3, No.6, December 2012

[11] Chunghoon Kim, Matthew Truk (2012), “A New Biased Discriminant Analysis Using Composite Vectors for Eye Detection”, IEEE Transaction on System Man and Machine

[12] Zhi-Hua Zhou, Xin Geng, "Projection Functions for Eye Detection”

[13] M. Hassabalth , Shun Ido (2009) “Eye Detection Using Intensity and Appearance Information”, Conference on Machine Vision Applications May 20-22, 2009 Yokohama, Japan

[14] D.M. Tasi, C.T. Lin "Fast normalized Cross-Correlation for Defect Detection"

[15] P. Lewis, "Fast Normalized Cross-Correlation" Industrial Light and Magic

[16] Dan Pei ZHAO, Hao FENG, Zhi guo JIANG (2009) “ A New Method of Multi-Scale Image Matching and Application Based on Fourier Phase Correlation”, Proceedings of SPIE Vol. 7495.

[17] Yehu Shen "Efficient normalized Cross-Correlation Calculation Method for Stereo Vision Based Robot Navigation.” ,Higher Education Press and Springer-Verlag

[18] Nuno Roma, Jose Santos-Victor, Jose Tome, "A Comparative Analysis of Cross-Correlation Matching Algorithm Using a Pyramidal Resolution Approach

[19] Chufs Face Database http://mmlab.ie.cuhk.edu.hk/facesketch.html.

[20] X. Wang and X. Tang. Face Photo-Sketch Synthesis and Recognition. IEEE Transactions on Pattern Analysis and Machine Intelligence (PAMI), vol. 31, no. 11, pages 1955-1967, 2009.

[21] W. Zhang, X. Wang and X. Tang. Coupled Information-Theoretic Encoding for Face Photo-Sketch Recognition. Proceedings of IEEE Conference on Computer Vision and Pattern Recognition (CVPR), 2011.

\section{Authors}

N K Bansode, research scholar in the Department of Computer Engineering, College of Engineering Pune. His areas of interest includes Evolutionary Computing, Computer Graphics and , Image Processing..

Dr P K Sinha, (IEEE Fellow and ACM Distinguished Engineer) is working as a Senior Director (High Performance Computing) working in the Centre for Development of Advanced Computing (CDAC), Pune. 